\title{
Anti-Inflammatory Potential of Liraglutide, a Glucagon-like peptide-1 receptor agonist, in Rats with Peripheral Acute Inflammation
}

İrem Mert ( $\sim$ irremmert@gmail.com )

Bolu Abant Izzet Baysal Universitesi Tip Fakultesi https://orcid.org/0000-0003-0501-5677

Ayhan Cetinkaya

Bolu Abant İzzet Baysal Üniv

Mujgan Gurler

Bolu Abant İzzet Baysal Univ

Canan Turel

Bolu Abant Izzet Baysal Univ

Humeyra Celik

Bolu abant Izzet Baysal

Ibrahim Ethem Torun

Bolu Abant Izzet Baysal

Idris Turel

Bolu Abant Izzet Baysal

\section{Research Article}

Keywords: Liraglutide, Tramadol, Hypersensitivity, Edema, Fever, Inflammation, Rat

Posted Date: October 25th, 2021

DOI: https://doi.org/10.21203/rs.3.rs-1003623/v1

License: (c) (i) This work is licensed under a Creative Commons Attribution 4.0 International License.

Read Full License

Version of Record: A version of this preprint was published at Inflammopharmacology on April 12th, 2022. See the published version at https://doi.org/10.1007/s10787-022-00978-0. 


\section{Abstract}

We tested our working hypothesis that that liraglutide (LRG), a Glucagon-like peptide-1 (GLP-1) receptor agonist, may potentiate the efficacy of tramadol (TR), a synthetic opioid, in acute inflammatory process and may has profound therapeutic effects in controlling the local acute inflammation. The present study aimed to explore the possible anti-inflammatory actions of LRG, TR or their combination treatments by investigating the inflammatory signs such as pain hypersensitivity, edema and fever in carrageenan (CG)induced acute peripheral inflammation model in rats. The levels of several biomarkers for inflammatory status, angiogenesis and oxidative stress were also measured in inflamed tissues. CG induced inflammation in the paws of rats caused identified by hypersensitivities, redness, edema and fever. LRG, TR or LRG+TR significantly improved the mechanical and cold allodynia, and reduced the edema and fever. LRG dramatically suppressed the inflammatory signs when compared to those of TR. In addition, co-administration of TR and LRG resulted in further reduction of sensitivity to cold and mechanical stimuli. Efficacies of LRG, TR or LRG+TR on inflammatory induced reactions altered depending on inhibition rates in the biomarkers of inflamed paws. Consequently, the suppressive actions of TR, LRG or $T R+L R G$ combination in the inflammation induced hypersensitivities, edema and fever indicates that these drugs have significant anti-inflammatory potential with anti-hypersensitivities, anti-edema and antipyretic effects. LRG with anti-inflammatory actions may be a highly promising options for the management of inflammatory conditions or inflammatory related various diseases.

\section{Introduction}

While inflammation is defined as a defensive mechanism of body against any harmful factor, acute inflammation is the first response involving a series of vascular and cellular events [1, 2]. The degree and characteristics of the inflammatory responses depend on the nature and severity of the damaging effect. Inflammatory responses involve complex processes are often mediated by the production of inflammatory mediators such as cytokines chemokines and growth factors via various immune cells [3, 4].

Carrageenan (CG) induced local acute inflammation in the paws of rats is a well-known experimental model to investigate local inflammatory processes and the anti-inflammatory effects of drug treatments [4]. This experimental model is characterized by inflammatory signs, such as swelling, heat, redness and pain behavior $[4,5]$.

Steroid and non-steroidal anti-inflammatory drugs are frequently preferred in the management of inflammatory diseases [6]. However, in recent years, a number of experimental and clinical studies have reported that many different pharmacological treatments can be beneficial in the management of inflammatory conditions.

It has been reported that inflammatory conditions can cause an increase in opioid receptor formation and their transport to peripheral endings [7]. Previous papers conducted with the inflammation model have 
also suggested that $\mu$ opioid receptor agonists can cause more effective peripheral analgesia than agonists of the other opioid receptors [7-10]. Tramadol (TR) hydrochloride (1RS, 2RS)-2-

[(dimethylamino)methyl]-1-(3-methoxyphenyl)-cyclohexonal $\mathrm{HCl}$ ) is a synthetic $\mu$ opioid receptor agonist [9]. Experimental and clinical studies show that TR has antinociceptive, analgesic and anti-inflammatory effects with few side effects. It can exert its effects through both $\mu$-receptor-mediated and non-receptormediated mechanisms [8-10].

Several papers have indicated that glucagon-like peptide-1 (GLP-1) receptors have a widespread distribution and that action mechanism of GLP-1 analogs is also closely related to various pathophysiological processes such as inflammation in addition to regulating blood glucose levels [11]. GLP-1 analogues such as liraglutide (LRG) are clinically used in the management of diabetic patients due to their insulinotropic properties $[11,12]$. LRG is a polypeptide GLP-1 analog drug produced by recombinant DNA technology. While the half-life of natural GLP-1 is approximately 1-2 minutes, LRG, which binds more tightly to albumin due to its molecular feature and thus is more resistant to dipeptidyl peptidase-4 activity, has a longer half-life and reaches approximately 12-13 hours [13-15].

Although several studies have displayed that LRG may have systemic anti-inflammatory potential in addition to glucose-lowering effects, to our knowledge there is no study showing the possible actions of LRG on local inflammatory processes and inflammatory responses. In this study, we hypothesized that considering the widespread distribution of GLP-1 receptors and the possible anti-inflammatory activity of LRG, LRG may potentiate the efficacy of TR, a synthetic opioid, in acute inflammatory process. To test this hypothesis, the possible effects of alone and combined applications of LRG and TR (LRG+TR) on inflammatory signs in a local acute inflammation model in rats were explored by examining the inflammatory induced responses (pain, edema and heat) and analyzing of various biomarkers of paw tissue for inflammatory status, oxidative stress, and angiogenesis.

\section{Materials And Methods}

\section{Animals}

Adult Wistar albino rats (240-250 g, provided by Bolu Abant İzzet Baysal University experimental animals application and research center) were used in experiments. Animals were housed under standard conditions with ad libitum access to food and water under a standard $12 \mathrm{~h}$ light/dark regime at room temperature $\left(23 \pm 2^{\circ} \mathrm{C}\right)$. Bolu Abant Izzet Baysal University animal experiments local ethical committee, Turkey approved the animal procedures (number 2020/30). All experiments were made according to guidelines of International Association for the Study of Pain.

\section{Carrageenan-induced acute peripheral inflammation}

Acute peripheral inflammation by $\lambda$-carrageenan (lambda CG, Sigma, Germany; $100 \mu \mathrm{L}$ of $2 \%(\mathrm{wt} / \mathrm{vol})$ is highly sensitive to investigate the inflammatory signs. Following the baseline behavioral measurements, CG intraplantarly (I.pl.) injected (1-mL syringe and a 27-gauge needle) into the right hindpaw at the 
midline the heel and anteriorly to the base of the second or third toe. An equal volume of saline (vehicle) was injected (I.pl) into the other hindpaw of rats for the control studies.

\section{Drugs and experimental groups}

In this present study, considering the previous studies using similar experimental models, and after power analysis $(a=0.05$, power $\geq 0.80), 6(n)$ animal studies were performed for each experimental group.

TR (Sigma-Aldrich Chemie GmbH, Munich, Germany) or LRG (Novo NordiskA/S, Bagsvaerd, Denmark) was administered to rats in $200 \mu \mathrm{l}$ saline solution at $1 \mathrm{mg} / \mathrm{kg}$ intraperitoneally (IP) 1 hour after CG injection. The dose of $1 \mathrm{mg} / \mathrm{kg}$ for both drugs was chosen based on our pilot and previous studies, together with published literature demonstrating the acute effects of these drugs. In TR+LRG combination treatments, $1 \mathrm{mg} / \mathrm{kg}$ (in $200 \mu \mathrm{l}$ saline) dose of both drugs was applied together.

Possible anti-inflammatory effects of systemically (IP) treated TR, LRG and their combination were investigated comparatively in localized inflamed paw (I.pl. CG injected right paw) and non-inflamed paw (I.pl. saline injected left paw) of rats in this present study. In the experiments, recordings from the untreated paw (non-inflamed left paw) in rats were used to compare the effect of drugs. The data to be obtained before drug administration were evaluated as control data in order to determine drug efficacy. Each experimental group $(n=6)$ was contained of two rat cages.

\section{Sensory Testing Procedure}

The animals were accustomed for at least $1 \mathrm{~h}$ to the experimental environments and were handled by the experimenter for 3 consecutive days before testing. The experimenter was blinded to the treatments of the rats until the experiments were completed.

Experiments were carried out at $23-24^{\circ} \mathrm{C}$ room temperature, in a quiet environment, between $9-16$ hours. Every effort was made to minimize the number of rats used and to alleviate their suffering during the experiments, which were carried out with due regard for ethical considerations. In this study, the effects of drug administration were examined 1 hour before, 1 hour after CG injection, and 5 hours after CG injection to limit the exposure time of rats and observe optimum inflammation induced responses.

\section{Von Frey Test}

The development of mechanical or tactile allodynia was evaluated with testing of mechanical withdrawal thresholds by applying calibrated Von Frey filaments (4-60 g) (Stoelting, Wood Dale, IL) [16].

Rats were placed on a metal mesh floor in transparent boxes separated by individual opaque separators. Filaments were applied vertically for 2 seconds until they were bent semicircularly to the plantar surface of both hind paws. If no response was received, a larger diameter filament was likewise applied. The filaments were applied to the mid plantar area 5 times in ascending order until paw withdrew, which was considered a positive response. The filament with which the paw withdrew was recorded as the threshold value. This withdrawal threshold was determined twice at 2-minute intervals and was used as the mean 
withdrawal threshold for data analysis. During the testing, the rat was considered to have an intense mechanical nociceptive sensory blockade when no response was observed using the filament with the largest diameter. Even with the lowest stimulus, it was considered the development of a sign of tactile allodynia if the rats withdrew their paws.

\section{Cold Plantar Test}

Cold plantar test was performed to evaluate the development of cold allodynia in rats [17]. Rats were placed in clear boxes separated by opaque separators on a $3 \mathrm{~mm}$ thick glass plate. The cold application probe was made from a modified $5 \mathrm{~mL}$ syringe filled with freshly powdered dry ice. After the dry ice was pelleted and the surface was flattened, the cold probe was applied to the hindpaw of the rat from under the glass. The cold probe induced a cooling ramp in the rat's paw in the temperature range of $5-10^{\circ} \mathrm{C}$ and elicited paw withdrawal responses. The withdrawal latency was measured with a chronometer.

Withdrawal latency for each rat was taken as the average of 3 trials at intervals of at least 2 minutes. Paw withdrawal latency is defined as the time it takes for rats to shake or withdraw their paw from the cold probe.

\section{Paw temperature measurement}

Thermal changes in the paws are used to determine the anti-inflammatory activities of the inflammatory process and applications, especially in the inflammation model. Temperature changes were evaluated by measuring the temperature of the inflamed and non-inflamed paws with an infrared thermometer $(2 \mathrm{~cm}$ from the middle surface of the paw). Data were obtained on the local antipyretic effects of the treatments.

\section{Evaluation of edema activity}

Intense edema occurs after CG injection to the paws of rats. Changes in paw masses were used to determine the possible anti-edema activities of the treatments. Paw masses of rats were measured at the end of the experiments. After scarification, paw edema was determined by measuring the mass $(\mathrm{g})$ of the paws cut from the ankle joint.

\section{Determination of the biomarkers in paw tissue}

At the end of experiments, animals were sacrificed under anesthesia and paw tissue samples were quickly removed with hygienic vehicles on ice and were kept at $-80^{\circ} \mathrm{C}$ until analysis. On the test day, the tissues were homogenized with phosphate buffer at a ratio of $1 / 9(0.1 \mathrm{~g}$ tissue: $0.9 \mathrm{ml} 50 \mathrm{mmol}$ phosphate buffer $\mathrm{pH}: 7.40$ ), and then centrifuged at $7000 \mathrm{rpm}$ for 5 minutes. Supernatants formed after centrifugation were studied using commercial kits.

\section{Measurements of Total Antioxidant Status and Total Oxidant Status}

TAS and TOS levels were measured using commercially available kits (Relassay, Turkey). 
The automated method for TAS level is based on the bleaching of characteristic color of a more stable ABTS (2,2' - Azino-bis(3-ethylbenzothiazoline-6-sulfonic acid)) radical cation by antioxidants. The results were expressed as mmol Trolox equivalent/L [18].

To measurement of TOS levels, oxidants present in the sample oxidized the ferrous ion-o-dianisidine complex to ferric ion. The oxidation reaction was enhanced by glycerol molecules abundantly present in the reaction medium. The ferric ion produced a colored complex with xylenol orange in an acidic medium. The color intensity, which could be measured spectrophotometrically, was related to the total amount of oxidant molecules present in the sample. The assay was calibrated with hydrogen peroxide and the results were expressed in terms of micromolar hydrogen peroxide equivalent per liter $(\mu \mathrm{mol} \mathrm{H} 2 \mathrm{O} 2$ equivalent/L) [19].

\section{Measurements of VEGF-A and TGF- $\beta 1$ )}

Sandwich enzyme-linked immunosorbent assay (ELISA) based kits used for the measurements of VEGFA or TGF- $\beta 1$ (Elabscience Biotechnology Co. Wuhan, China). Standards or samples are added to the micro ELISA plate wells and combined with the specific antibody. Then a biotinylated detection antibody specific for universal VEGF-A or TGF- $\beta 1$ and Avidin-Horseradish Peroxidase (HRP) conjugate are added successively to each micro plate well and incubated. Free components are washed away. The substrate solution is added to each well. Only those wells that contain universal VEGF-A or TGF- $\beta 1$, biotinylated detection antibody and Avidin-HRP conjugate will appear blue in color. The enzyme-substrate reaction is terminated by the addition of stop solution and the color turns yellow. The optical density (OD) is measured spectrophotometrically at a wavelength of $450 \mathrm{~nm} \pm 2 \mathrm{~nm}$. The OD value is proportional to the concentration of universal VEGF-A or TGF- $\beta 1$.

\section{Measurements of TNF- $a$ and IL-10}

Sandwich enzyme-linked immunosorbent assay (ELISA) based kits used for the measurements of TNF-a and IL-10 (Bioassay Technology Laboratory (BT Lab), Inc., Shanghai, China). TNF-a or IL-10 present in the sample is added and binds to antibodies coated on the wells. And then biotinylated Rat TNF-a or IL-10 antibody is added and binds to TNF-a or IL-10 in the sample. Then streptavidin-HRP is added and binds to the biotinylated TNF-a or IL-10 antibody. After incubation unbound streptavidin-HRP is washed away during a washing step. Substrate solution is then added and color develops in proportion to the amount of Rat TNF- $a$ or IL-10. The reaction is terminated by addition of acidic stop solution and absorbance is measured at $450 \mathrm{~nm}$.

\section{Statistical analyses}

Findings were presented as mean \pm standard deviation (SD). All statistical analyses were performed using analysis of variance (ANOVA, Statistical Package for Social Sciences 15.0, SPSS, Chicago, IL, USA). A one or two-way repeated-measures of ANOVA with Bonferroni's post hoc test was used to compare treatments. $\mathrm{P}<0.05$ was considered significant. 


\section{Results}

While experiments in inflamed right paw were conducted to identify possible anti-inflammatory actions of drugs, and findings from non-inflamed left paw were used to identify possible changes in the sensor sensitivity.

It was determined that the basal cold latency was $21.0 \pm 3.5 \mathrm{~s}$, and the basal mechanical threshold value was $26.0 \pm 0.5 \mathrm{~g}$ before the CG injection (Fig. 1 and 2). These values did not significantly different between the inflamed and non-inflamed paws. I.pl. injection of saline for $\mathrm{CG}$ control did not change the basal threshold or latency values.

\section{Changes in mechanical thresholds after the treatments}

Carrageenan significantly decreased the threshold value (Fig. 1B). CG-induced reduction in the threshold indicates the development of mechanical allodynia in rats. The mechanical allodynic response, which appeared 1 hour after $C G$ injection, reached its highest level after 5 hours $(P<0.05)$.

TR, LRG or TR+LRG treatments caused significant enhancements in the decreased threshold by CG $(P<0.05)$. While $L R G$ increased the threshold more than TR, the increases in the threshold values reached the highest level in the TR+LRG combined treatment $(P<0.05)$ (Fig. 1B).

Responses to mechanical stimuli from the non-inflamed (left) paw were used to evaluate the effects of the treatments on tactile sensation of rats (Fig. 1A). Saline administration (control for drug) did not cause a significant change in threshold, while treatments of drugs significantly decreased the tactile sensitivity 4 hours after administration $(P<0.05)$. LRG increased the threshold more than that of TR $(P<0.05)$. The TR+LRG combination increased the threshold even more $(P<0.05)($ Fig. 1A).

\section{Changes in cold latency after the treatments}

Paw withdrawal latency to cold stimulus significantly reduced after CG injection. This decrease indicates the development of cold allodynia in rats. CG-induced cold allodynia peaked after 5 hours (Fig. 2B).

TR, LRG or TR+LRG treatments caused significant increases in the decreased latency by $C G(P<0.05)$. LRG increased the withdrawal latency more than that of $T R(P<0.05)$. However, when $L R G$ and $T R+L R G$ administrations were compared, it was found that the latency values did not statistically different( $P>0.05)$ (Fig. 2B).

Responses to cold stimuli from the non-inflamed (left) paw were used to evaluate the effects of the treatments on cold sensation of rats (Fig. 2A). Saline administration (IP) did not change the latency, while all treatments significantly decreased the cold sensitivity 4 hours after administration. LRG caused a greater increase in latency compared to TR, while the combination of TR+LRG increased latency even more $(P<0.05)$ (Fig. 2A).

\section{Effects of treatments in paw temperature}


In the study, paw temperatures were measured with an infrared thermometer 5 hours after the CG injection (4 hours after the drug administration), to determine the possible anti-pyretic effects of the drugs. Temperature significantly increased in the CG-injected paw $\left(33.6 \pm 1.7^{\circ} \mathrm{C}\right)$ compared to the contralateral non-inflamed paw (saline injected) $\left(26.6 \pm 1.1^{\circ} \mathrm{C}\right)(\mathrm{P}<0.05)$ (Fig. 3A). Although TR decreased the increase in paw temperature by the inflammation, the change did not statistically significant $(P>0.05)$. While the LRG and TR+LRG combination significantly suppressed the increase in paw temperature, there was no significant difference between the results of the two treatments (Fig. 3A). These results are important in terms of showing the anti-pyretic activities of LRG and TR+LRG combination, despite the partial anti-pyretic activity of TR. However, all treatments did not significant change in temperature of non-inflamed paws.

\section{Effects of treatments in paw mass}

In order to evaluate the anti-edema effects of the drugs, the paw masses were measured 5 hours after the CG injection (4 hours after the drug administration). When compared to the non-inflamed contralateral paw mass $(1.47 \pm 0.13 \mathrm{~g})$, CG-treated paw mass significantly increased $(2.54 \pm 0.31 \mathrm{~g})(\mathrm{P}<0.05)$ (Fig. 3B). $T R$, LRG or TR+LRG combination treatments significantly suppressed the increase in paw mass $(P<0.05)$. These results are important in terms of demonstrating the anti-edema efficacy of TR, LRG and TR+LRG combination. When compared the anti-edema actions of the all drug treatments, there were no statistically significant differences. However, all treatments did not significant changes in paw masses of non-inflamed contralateral paws.

\section{Biochemical Analysis}

TOS level significantly increased in the paw tissue after $C G$ induced inflammation $(P<0.05)$ (Fig. 4B). However, TR, LRG and TR+LRG combination treatments did not statistically insignificant changes in the increased TOS level of inflamed paw tissue. TAS level did not significantly change in the inflamed paw tissue. While the TR application did also not cause any significant change in the TAS level, LRG application caused a significant increase in TAS level of inflamed paw $(P<0.05)$ (Fig. 4A). TR+LRG combination application caused a statistically insignificant increase in TAS level $(P<0.05)$.

TNF-a levels significantly enhanced in the inflamed paw tissue $(P<0.05)$ (Fig. 5A). While TR significantly suppressed this increase TNF-a level $(P<0.05)$, the combination of $L R G$ and $T R+L R G$ did not cause any significant change. IL-10 level significantly increased in inflamed paw tissue when compared to noninflamed paw $(P<0.05)$ (Fig. 5B). While the increased IL-10 level in inflamed paw tissue did not change after TR application, LRG or TR+LRG combination significantly increased the IL-10 level $(P<0.05)$.

VEGF level significantly increased in the inflamed paw tissue of rats compared to the non-inflamed paw $(P<0.05)$. Although not statistically significant, TR showed a tendency to suppress the VEGF level in inflamed tissue. LRG or combination of TR and LRG resulted in statistically significant reductions in tissue VEGF level $(P<0.05)$ (Fig. 6A). TGF- $\beta 1$ levels also significantly increased in the inflamed paw tissue 
of rats $(P<0.05)$ (Fig. 6B). While TR did not cause a significant change in TGF- $\beta 1$ level, $L R G$ or TR+LRG combination significantly suppressed TGF- $\beta 1$ level in inflamed paw tissue $(P<0.05)$.

All treatments did not cause any significant changes in the levels of biomarkers mentioned above in noninflamed contralateral paws.

\section{Discussion}

The findings showed the anti-hypersensitive efficacies of systemically administered TR, LRG or TR+LRG combination in reducing mechanical and cold allodynia caused by CG injection in the rat paw. In addition, TR, LRG and TR+LRG combination suppressed the CG-induced rat paw edema and paw temperature increase, even at different levels, indicating that these treatments have significant anti-inflammatory potential, together with their anti-edema and anti-pyretic effects. Finding also suggested that decreases in tactile and cold sensitivities by these treatments in the non-inflamed paw may indicate their anesthetic/hypoesthetic potential. To the best of our knowledge, this is the first report showing the antihypersensitive, anti-edema and anti-pyretic actions of LRG in rats with acute peripheral inflammation.

In this present study, development of edema, redness and inflammatory pain behaviors, which are indicators of inflammatory reaction, were observed within the first hour following CG injection. The development of allodynia, which is defined as a painful response to a stimulus that does not produce pain responses [20,21], was observed after mechanical and cold stimuli. Sensor tests were performed 3 times within 5 hours ( 1 hour before, 1 hour and 5 hours after CG injection) and the results were evaluated for inflammatory pain behaviors. Mechanical and cold allodynia responses peaked 5 hours after CG injection. In addition, remarkable edema and temperature increase were detected in the paw. In addition, responses to mechanical and cold stimuli from the non-inflamed paw were used to evaluate the effects of the treatments on the tactile and cold sensitivities of rats, and to determine possible anesthetic (hypoesthetic) efficacies.

Inflammatory pain hypersensitivity is the cardinal sign of acute peripheral inflammation that occurs depending on the process $[20,21]$. Consistent with previous works, decreases in mechanical threshold and cold latency values is an indication of the development of allodynia (mechanical and cold hypersensitivities) [20-22]. It has been known that sensory afferents contribute to inflammatory pain hypersensitivity. Peripheral inflammation may cause increases in the sensitivities of the peripheral nerve terminals of A-delta and $C$ nerve fibers at the inflammation site $[1,5,23]$.

The effects of TR and LRG on latency and threshold parameters were different from each other. TR enhanced the threshold and latency in inflamed rats, while mechanical and cold sensitivity in the noninflamed paw were reduced by TR treatment. Findings, as expected, showed that TR may has antihypersensitive and anesthetic effects. These results are consistent with the data obtained from previous studies $[9,10]$. It has been previously reported that TR may produce analgesic/anesthetic activities due to its high affinity for $\mu$-opioid receptors as well as inhibition of both serotonin and norepinephrine reuptake 
[7-9]. There is also lots of evidence that TR inhibits the activity of voltage-gated $\mathrm{Na}$ channels, delayed rectifier $\mathrm{K}$ channels, $\mathrm{N}$-methyl-d-aspartate receptors, and substance $\mathrm{P}$ receptors in vitro $[8,10,24,25]$.

LRG (compared to TR) further increased the reduced latency and threshold values of rats with paw inflammation, revealing its potent anti-hypersensitive (anti-allodynic) activity. Moreover, LRG treatment also resulted in greater increases in both threshold and latency compared to TR in non-inflamed rats. In addition to the anti-hypersensitive effects of LRG in inflamed rats, the anesthetic/ hypoesthetic effects of LRG in non-inflamed rats showed that LRG may also modulate the conduction and function of sensory nerves which relay the peripheral information to the spinal cord. Previous studies have been reported that in addition to neuroprotective effect in diabetic neuropathy LRG treatments enhance the neurogenesis, recover the cognitive function, and decrease the amyloid plaque deposition in Alzheimer's disease models [26-28]. The present study reports for the first time the anti-hypersensitive potential of LRG in rats with peripheral acute inflammation.

When two drugs co-administered, the treatment can produce independent effects or additive effects (equal to the sum of the effects of each). Combined treatment can also inhibit or decrease each other's effects (antagonism), or, the effect can be greater than the expected effect (synergy). In this present study, combined administration of an opioid, TR, and an anti-diabetic, LRG, resulted in further reduction of sensitivity in both the inflamed and non-inflamed paw. The remarkable suppression of cold and mechanical allodynia with anti-hypersensitive activity may suggest that this combination treatment may be very effective in inflammatory pain.

Paw masses were measured at the end of the experiments ( 5 hours after CG application) to determine the anti-edema effects of the treatments. Increased edema activity following CG administration resulted in an increase in paw mass up to approximately 2 times after 5 hours. TR, LRG or TR+LRG combination treatments showed significant decreases on the paw masses at approximately the same levels. These results can show the anti-edema effects of TR, LRG and TR+LRG combination.

One of the most important signs of inflammation after CG was a significant increase in the temperature of the inflammation area [1-3]. The increase in temperature in inflammation is an indication that the cellular immune defense mechanisms are activated against the harmful factor, which is caused by different mediators released in the inflammation area. This increase in temperature of the inflammation area was significantly reduced by all drug treatments. However, compared to TR, LRG caused a greater reduction in the temperature of the inflammation site. These results may clearly demonstrate the antipyretic effects of TR, LRG or TR+LRG combination treatments.

The presented findings may imply that the anti-hypersensitive, antipyretic and anti-edema effects of treatments may be closely related with the inflammatory environments includes various mediators such as cytokines, chemokines, and growth factor proteins which, alone or altogether. In order to obtain more detailed information about the molecular action mechanisms of treatments, the changes of biomarkers in the inflammation site were determined. 
Findings showed that the levels of both pro-inflammatory and anti-inflammatory cytokines may increase in inflammation area. It has been known that pro-inflammatory cytokines released from inflammatory cells coming to the inflammation area after the inflammatory factor may cause more leukocyte migration to the area and cause an increase in pro-inflammatory cytokines [3,4]. The main function of antiinflammatory cytokines is to inhibit or suppress the effect produced by pro-inflammatory cytokines and thus to maintain the balance between pro- and anti-inflammatory cytokines [3]. While TR produced the anti-inflammatory activity by suppressing the increase of the pro-inflammatory cytokine TNF-a, the treatments of LRG or LRG+TR combination showed anti-inflammatory activity by increasing the level of anti-inflammatory cytokine IL-10.

Angiogenesis is a biological event that plays a role in many physiological and pathological processes [29]. VEGF is one of the most important factors stimulating angiogenesis, and its inhibition can also be selected as a therapeutic target to reduce angiogenesis $[29,30]$. It is known that TGF- $\beta 1$ increases the release of VEGF angiogenic factors and is important in the occurrence of complications. In this present study, the suppression of VEGF-A and TGF- $\beta 1$ levels by LRG suggests that the anti-angiogenic property of LRG may be an important mechanism contributing to its anti-inflammatory activity.

Many previous studies have shown that oxidative stress can play important roles in the inflammatory process [31, 32]. In this study, it was found that TAS did not change in inflamed paw tissues, while TOS increased significantly. While TR did not cause any change, LRG and LRG +TR increased the TAS level in inflamed tissue. The findings may suggest that LRG treatment may be important for the prevention of inflammation induced oxidative stress due to its anti-oxidant properties.

Consequently, compared with TR, which known effects, reduction of sensitivities to sensory stimuli of the non-inflamed paw by LRG treatment may indicate that it has anesthetic/hypoesthetic effects, which are described by reduced or slowing of sensory nerve conduction and functions. LRG may also ameliorate the pain hypersensitivity, edema and fewer. Anti-hypersensitive, anti-edema and antipyretic effects of LRG in rats with peripheral acute inflammation may be due to its anti-inflammatory, anti-angiogenic and antioxidative actions tissues (Fig. 7). Furthermore, LRG, when combined with TR, may be an important treatment option for controlling inflammation and inflammatory signs. However, more experimental and molecular studies are needed, especially to examine the anti-inflammatory potential of LRG and its mechanisms of action in the underlying inflammatory processes. In addition, studies on the pharmacological mechanisms of the additive/synergistic effect occurring in the combined application of LRG and TR are required.

\section{Declarations}

\section{AUTHOR'S CONTRIBUTION}

I.M., A.C, M.G., C.T., H.C., I.E.T. and I.T. participated in the design, experiments, analysis, and drafting of the manuscript. I.M and A.C supervised the study. 


\section{FUNDING}

This study was supported by the Bolu Abant Izzet Baysal University Scientific Research Fund (Grant number 2021.08.36.1497)

\section{DATA AVAILABILITY}

Not applicable

\section{Ethical Approval}

All mice studies were conducted according to protocols approved by Bolu Abant Izzet Baysal University animal experiments local ethical committee, Turkey with the approval number number 2020/30.

\section{Consent for Publication}

All authors have reviewed the manuscript and have given consent for publication.

\section{Competing Interests}

The authors declare no competing interests.

\section{References}

1. Libby, P. 2007. Inflammatory mechanisms: the molecular basis of inflammation and disease. Nutrition Reviews 65: 140-146.

2. Zhang, J.M., and J. An. 2007. Cytokines, inflammation, and pain. International Anesthesiology Clinics 45: 27-37.

3. Dawes, J.M., and S.B. McMahon. 2013. Chemokines as peripheral pain mediators. Neuroscience Letters 557: 1-8.

4. Annamalai, P., and E.B..Thangam. 2017. Local and systemic profiles of inflammatory cytokines in carrageenan-induced paw inflammation in rats. Immunological Investigations 46: 274-283.

5. Barrot, M. 2012. Tests and models of nociception and pain in rodents. Neuroscience 211: 39-50.

6. Rao, P., and E.E. Knaus. 2008. Evolution of nonsteroidal anti-inflammatory drugs (NSAIDs): cyclooxygenase (COX) inhibition and beyond. Journal of Pharmacy and Pharmaceutical Sciences 11(2):81-110.

7. Stein, C., and C. Zöllner. 2009. Opioids and sensory nerves. Handbook of Experimental Pharmacology (194): 495-518.

8. Reeves, R.R., and R.S. Burke. 2008. Tramadol: basic pharmacology and emerging concepts. Drugs Today 44: 827-836.

9. Raffa, R.B., E. Friderichs, W. Reimann, R.P. Shank, E.E. Codd, and J.L. Vaught. 1992. Opioid and non opioid components independently contribute to the mechanism of action of tramadol, an 'atypical 
opioid analgesic. Journal of Pharmacology and Experimental Therapeutics; 260: 275-278.

10. Mert, T., Y. Gunes, and I. Gunay. 2007. Local analgesic efficacy of tramadol following intraplantar injection. European journal of Pharmacology 558: 68-72.

11. Cho, Y.M., R.D. Wideman, and T.J. Kieffer. 2013. Clinical application of glucagon-like Peptide 1 receptor agonists for the treatment of type 2 diabetes mellitus. Endocrinology and Metabolism (Seoul) 28(4): 262-274.

12. Calsolaro, V., and P..Edison. 2015. Novel GLP-1 (Glucagon-Like Peptide-1) Analogues and Insulin in the Treatment for Alzheimer's Disease and Other Neurodegenerative Diseases. CNS Drugs 29(12): 1023-1039.

13. Hölscher, C. 2012. Potential role of glucagon-like peptide-1 (GLP-1) in neuroprotection. CNS Drugs 26(10): 871-882.

14. Tang, S.T., Q. Zhang, H.Q. Tang, C.J. Wang, H. Su, and Q. Zhou. 2016. Effects of glucagon-like peptide-1 on advanced glycation endproduct-induced aortic endothelial dysfunction in Streptozotocin-induced diabetic rats: Possible roles of rho kinase-and AMP kinase-mediated nuclear factor kappa signaling pathways. Endocrine; 53(1):107-116.

15. Meier, J.J. 2012. GLP-1 receptor agonists for individualized treatment of type 2 diabetes mellitus. Nature Reviews Endocrinology 8:728-742.

16. Chaplan, S.R., F.W. Bach, J.W. Pogrel, J.M. Chung, and T.L. Yaksh. 1994. Quantitative assessment of tactile allodynia in the rat paw. Journal of Neuroscience Methods 53:55-63.

17. Brenner, D.S., J.P. Golden, and R.W. Gereau. 2012. A novel behavioral assay for measuring cold sensation in mice. PLoS One 7:e39765.

18. Erel, O. 2004. A novel automated direct measurement method for total antioxidant capacity using a new generation, more stable ABTS radical cation. Clinical Biochemistry 37: 277-285.

19. Erel, O. 2005. A new automated colorimetric method for measuring total oxidant status. Clinical Biochemistry 38: 1103-1111.

20. Kidd, B.L., and L.A. Urban. 2001. Mechanisms of inflammatory pain. British Journal of Anaesthesia 87: 3-11.

21. Coutaux, A., F. Adam, J.C. Willer, and D. Le Bars. 2005. Hyperalgesia and allodynia: peripheral mechanisms. Joint Bone Spine 72(5):359-371.

22. ippoldt, E.K., S. Ongun, G.K. Kusaka, and D.D. McKemy. 2016. Inflammatory and neuropathic cold allodynia are selectively mediated by the neurotrophic factor receptor GFRa3. Proceedings of the National Academy of Sciences USA. 113(16):4506-4511.

23. Basbaum, A.I., D.M. Bautista, G. Scherrer, and D. Julius. 2009. Cellular and molecular mechanisms of pain. Cell 139:267-284.

24. Mert, T., Y. Gunes, D. Ozcengiz, I. Gunay, and S. Polat. 2006. Comparative effects of lidocaine and tramadol on injured peripheral nerves. European Journal of Pharmacology 543: 54-62. 
25. Hara, K., K.Minami, and T. Sata, 2005. The effects of tramadol and its metabolite on glycine, gammaaminobutyric acidA, and N-methyl-D-aspartate receptors expressed in Xenopus oocytes. Anesthesia and Analgesia. 100, 1400-1405.

26. Moustafa, P.E., N.F. Abdelkader, S.A. El Awdan, O.A. El-Shabrawy, and H.F. Zaki. 2018. Liraglutide ameliorated peripheral neuropathy in diabetic rats: Involvement of oxidative stress, inflammation and extracellular matrix remodeling. Journal of Neurochemistry 146(2): 173-185.

27. Parthsarathy, V., and C. Holscher. 2013. Chronic treatment with the GLP1 analogue liraglutide increases cell proliferation and differentiation into neurons in an AD mouse model. PLOS ONE 8, e58784.

28. Palleria, C., A. Leo, F. Andreozzi, R. Citraro, M. lannone, R. Spiga, G. Sesti, A. Constanti, G. De Sarro, F. Arturi, and E. Russo. 2017. Liraglutide prevents cognitive decline in a rat model of streptozotocininduced diabetes independently from its peripheral metabolic effects. Behavioural Brain Research 321: 157-169.

29. Ferrara, N. 2002. VEGF and the quest for tumour angiogenesis factors. Nature Reviews Cancer 2 : 795- 803.

30. Sporn, M.B., and A.M. Roberts. 1992. Transforming growth factor beta: recent progress and new challenges. Journal of Cell Biology 119: 1017-1021.

31. Halici, Z., G.O. Dengiz, F. Odabasoglu, H. Suleyman, E. Cadirci, and M. Halici. 2007. Amiodarone has antiinflammatory and anti-oxidative properties: an experimental study in rats with carrageenaninduced paw edema. European Journal of Pharmacology; 566(1): 215-221.

32. Conner, E.M., and M.B. Grisham. 1996. Inflammation, free radicals, and antioxidants. Journal of Nutrition 12(4): 274-277.

\section{Figures}




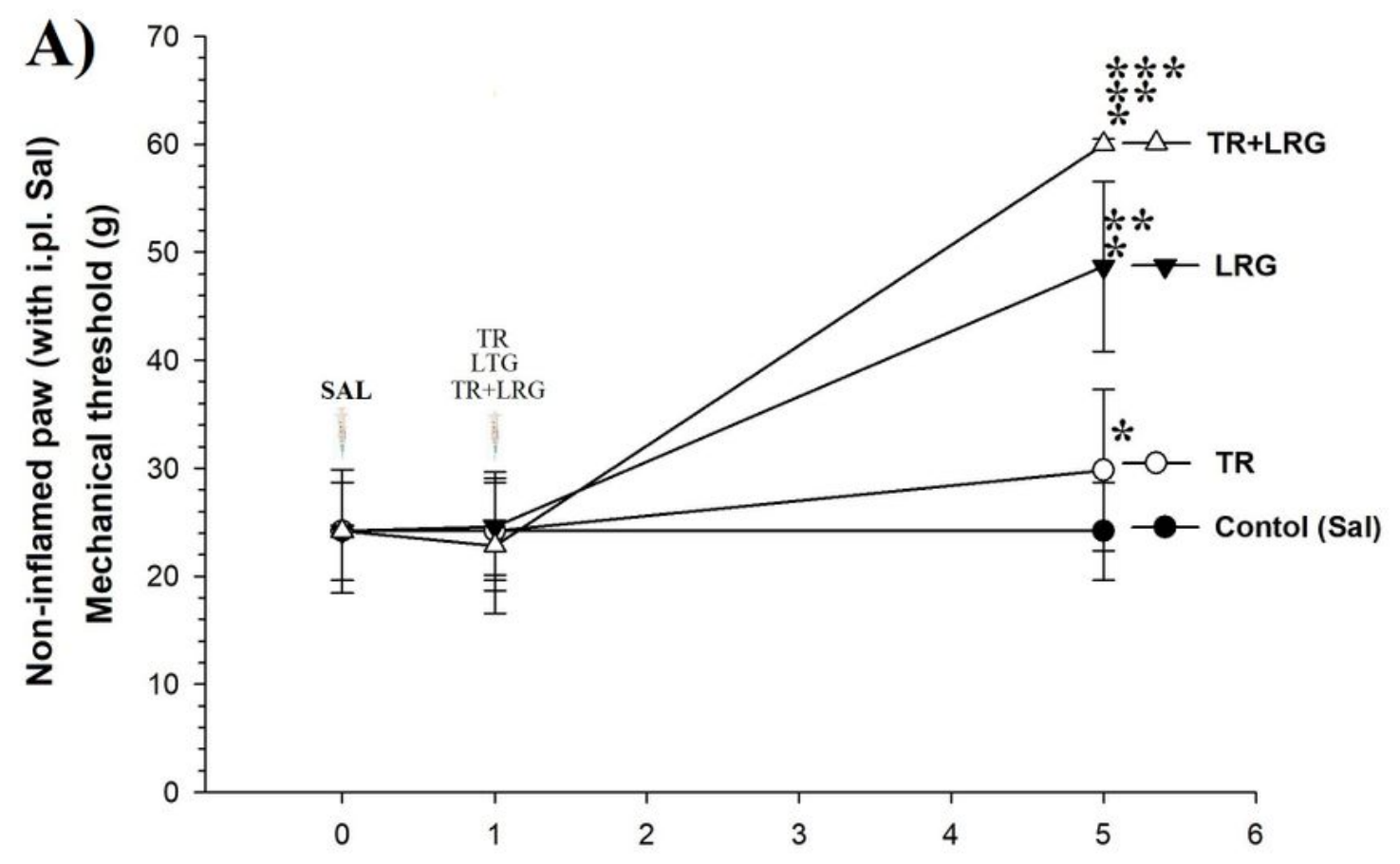

Time (h)

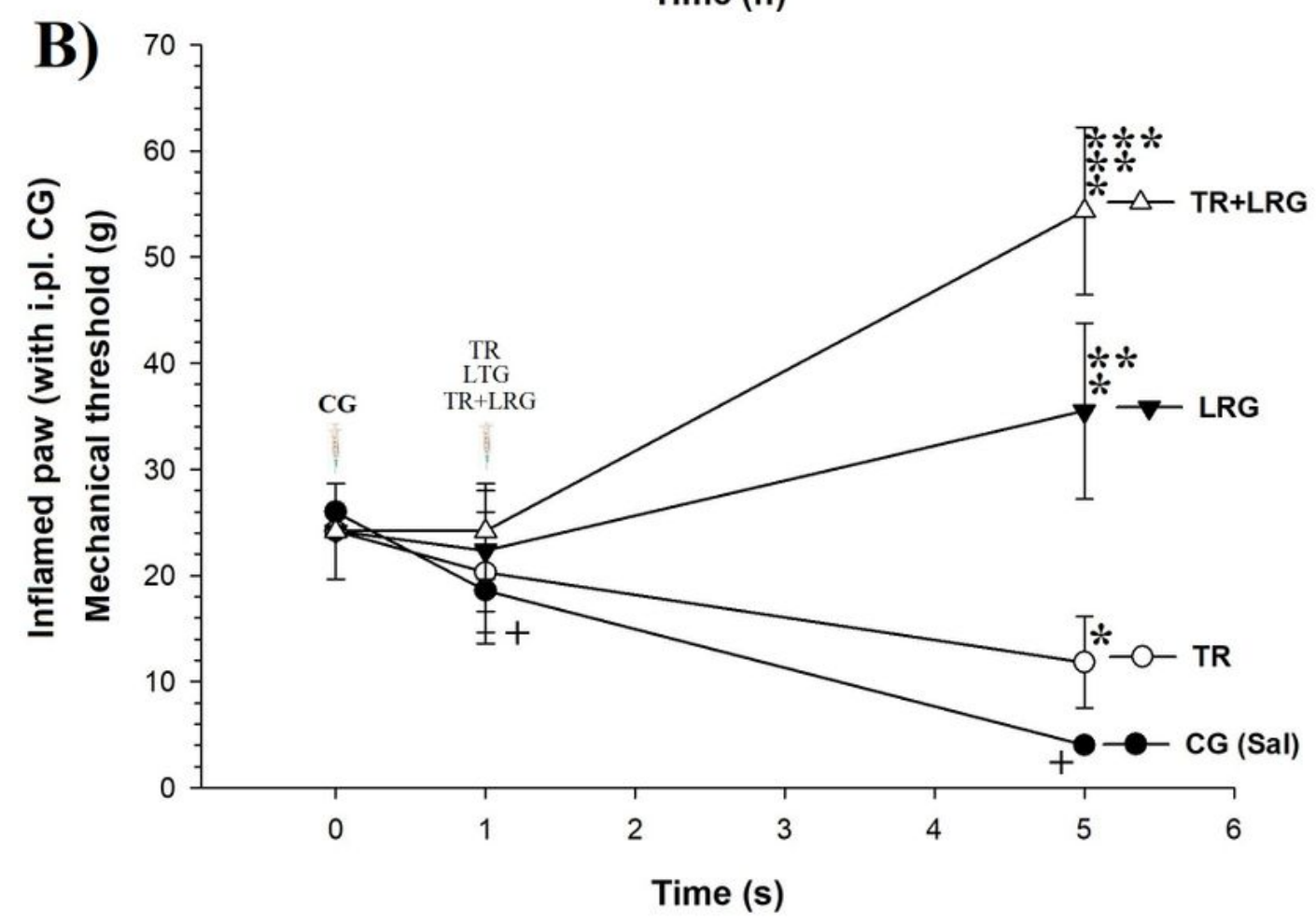

Figure 1

Changes in withdrawal mechanical thresholds in the non-inflamed $(A)$ and inflamed $(B)$ paws of rats. Tramadol (TR), Liraglutide (LRG) or their combination (TR+LRG) treated to rats $1 \mathrm{~h}$ after carrageenan (CG) or Saline (SAL) injections during the experiments. Each point represents the mean value of 6 rats, and the vertical bars indicate $\pm S D$. $+P<0.05$ indicates significant differences as compared to baseline value. * $\mathrm{P}<0.05$ indicates significant differences as compared to control group. ${ }^{* *} \mathrm{P}<0.05$ indicates significant 
differences as compared to TR group *** $P<0.05$ indicates significant differences as compared to LRG group

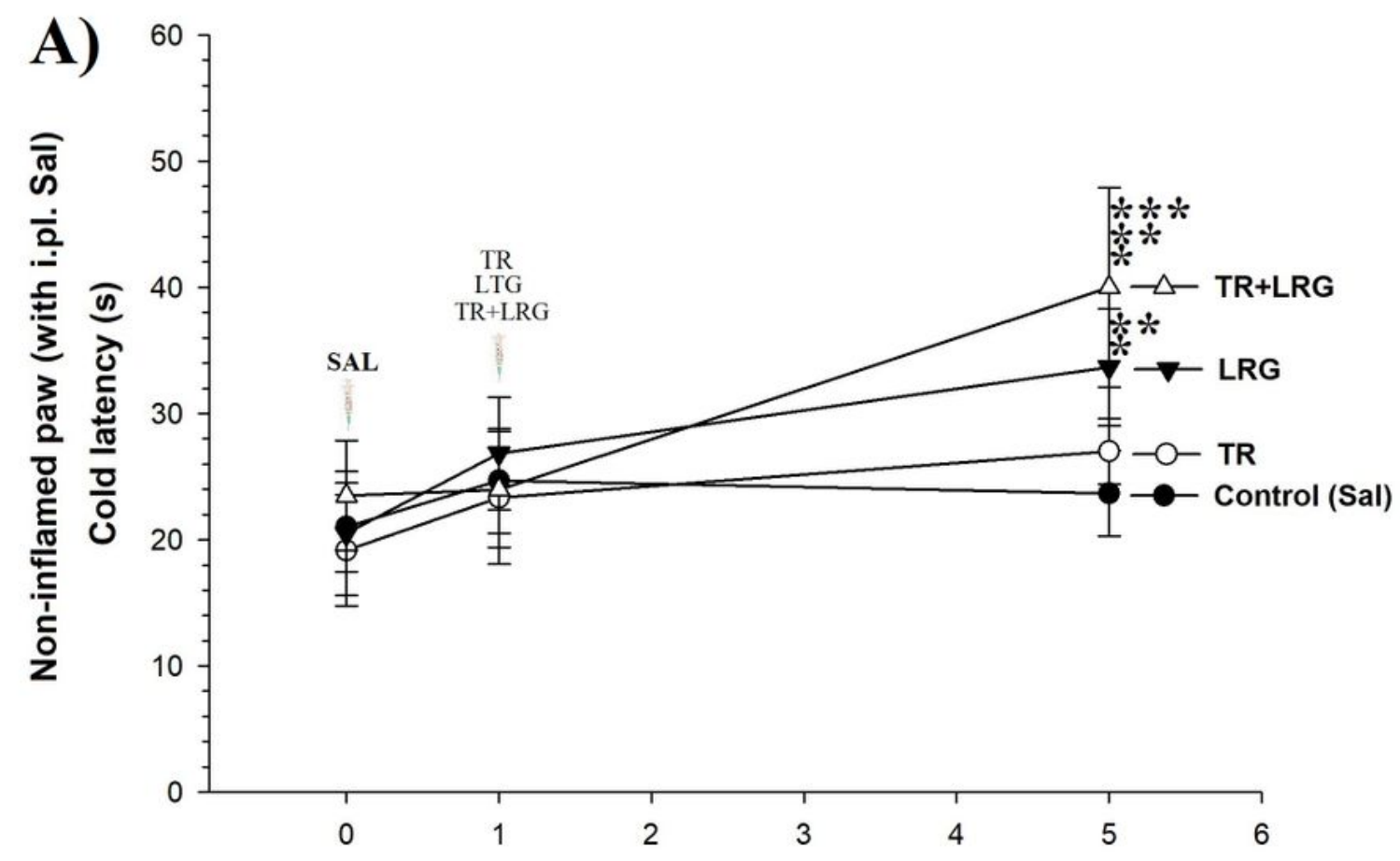

Time (s)

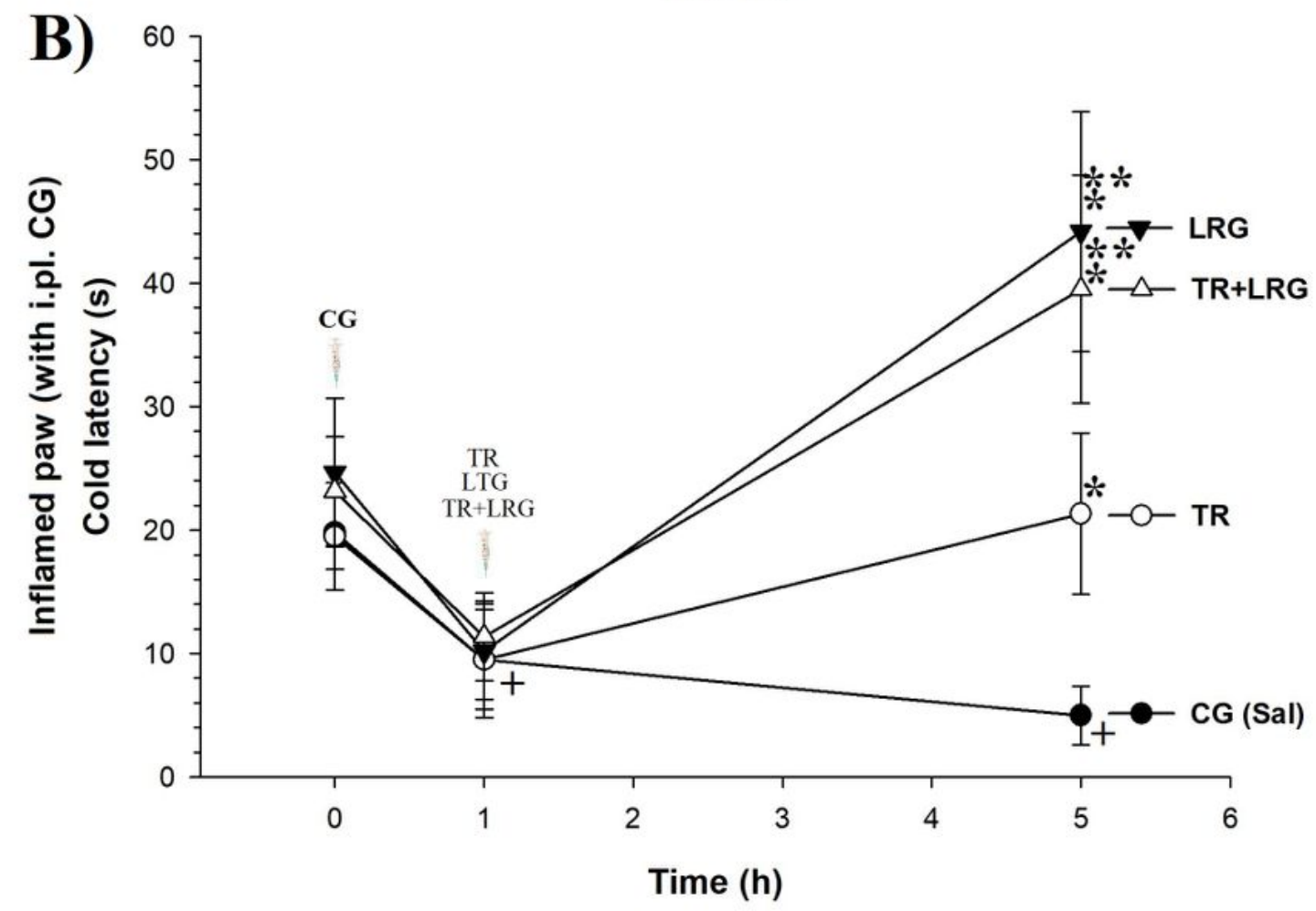

Figure 2

Changes in withdrawal cold latencies in the non-inflamed $(A)$ and inflamed $(B)$ paws of rats. Tramadol (TR), Liraglutide (LRG) or their combination (TR+LRG) treated to rats $1 \mathrm{~h}$ after carrageenan (CG) or Saline $(\mathrm{SAL})$ injections during the experiments. Each point represents the mean value of 6 rats, and the vertical 


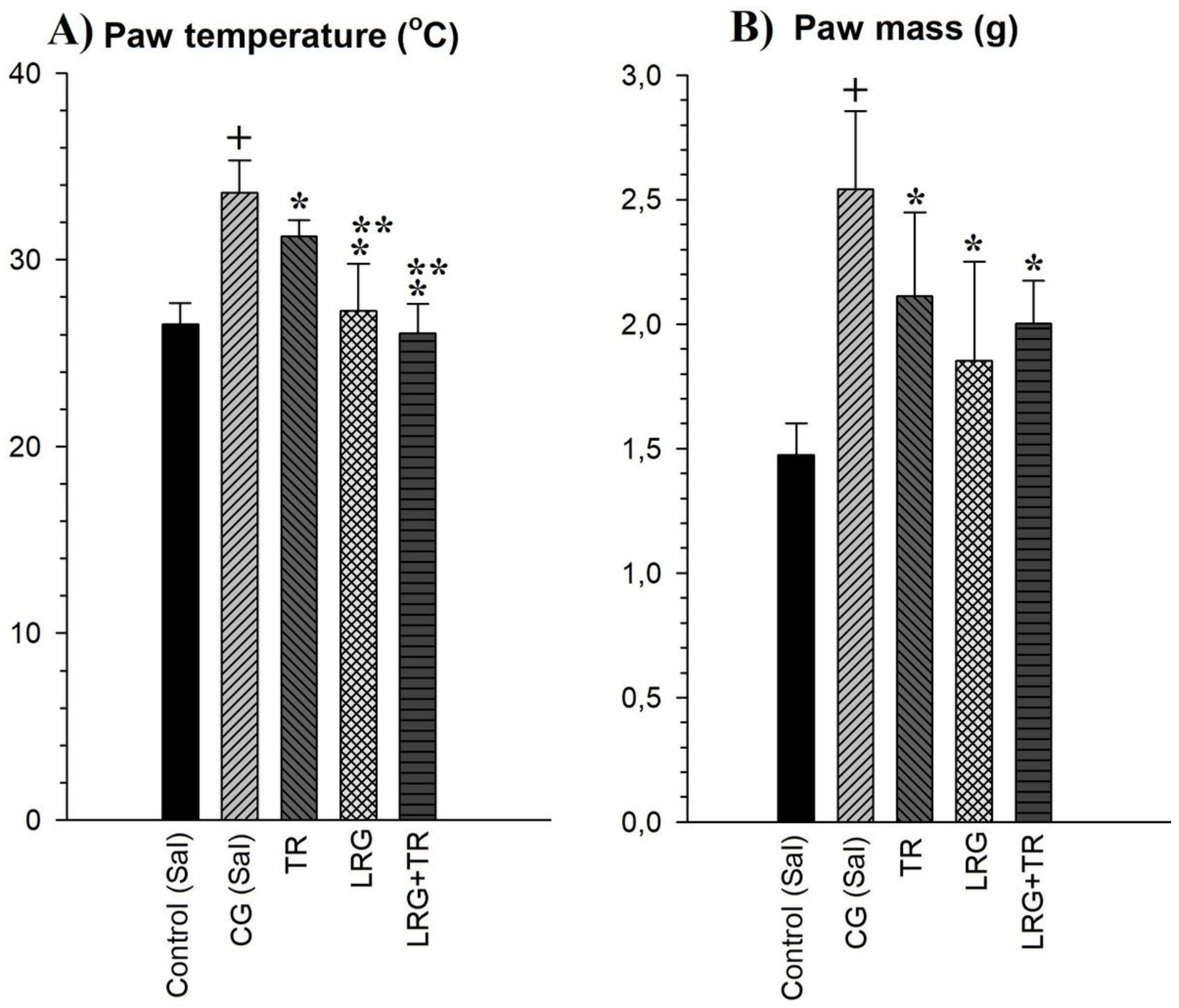

Figure 3

Changes in paw temperature for antipyretic activities (A) and paw masses for anti-edematous activities (B) of Tramadol (TR), Liraglutide (LRG) or their combination (TR+LRG) in carrageenan (CG) induced inflamed paws of rats. Each bar represents the mean value of 6 rats, and the vertical bars indicate \pm SD. + $P<0.05$ indicates significant differences as compared to control (saline) group. * $P<0.05$ indicates significant differences as compared to CG-control group. ${ }^{*} \mathrm{P}<0.05$ indicates significant differences as compared to TR group 

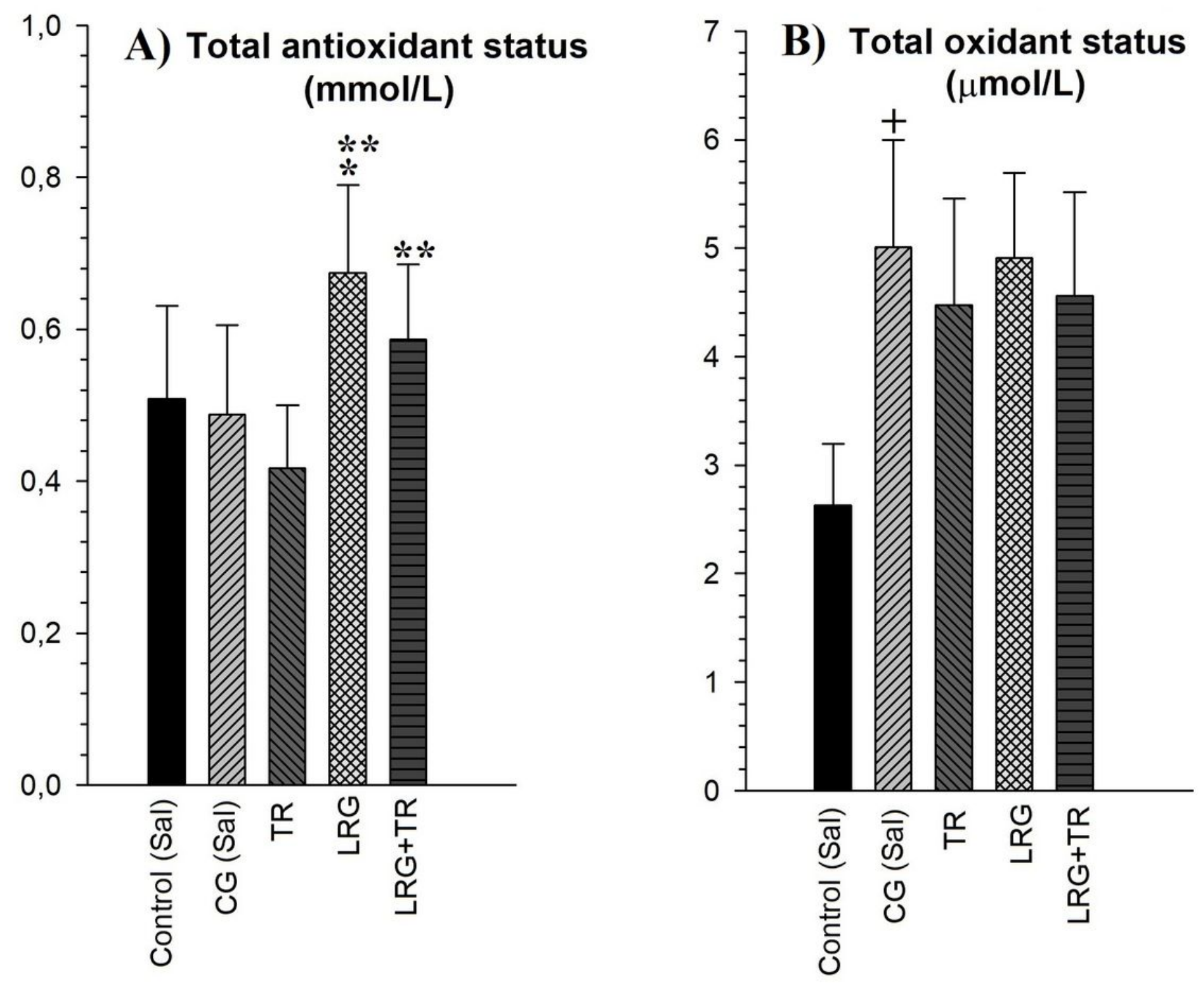

Figure 4

Effects of Tramadol (TR), Liraglutide (LRG) or their combination (TR+LRG) on total antioxidant status (TAS) (A) and total oxidant status (TOS) (B) in carrageenan (CG) induced inflamed paws of rats. Each bar represents the mean value of 6 rats, and the vertical bars indicate $\pm S D$. $+P<0.05$ indicates significant differences as compared to control (saline) group. * $P<0.05$ indicates significant differences as compared to CG-control group. ** $\mathrm{P}<0.05$ indicates significant differences as compared to TR group 

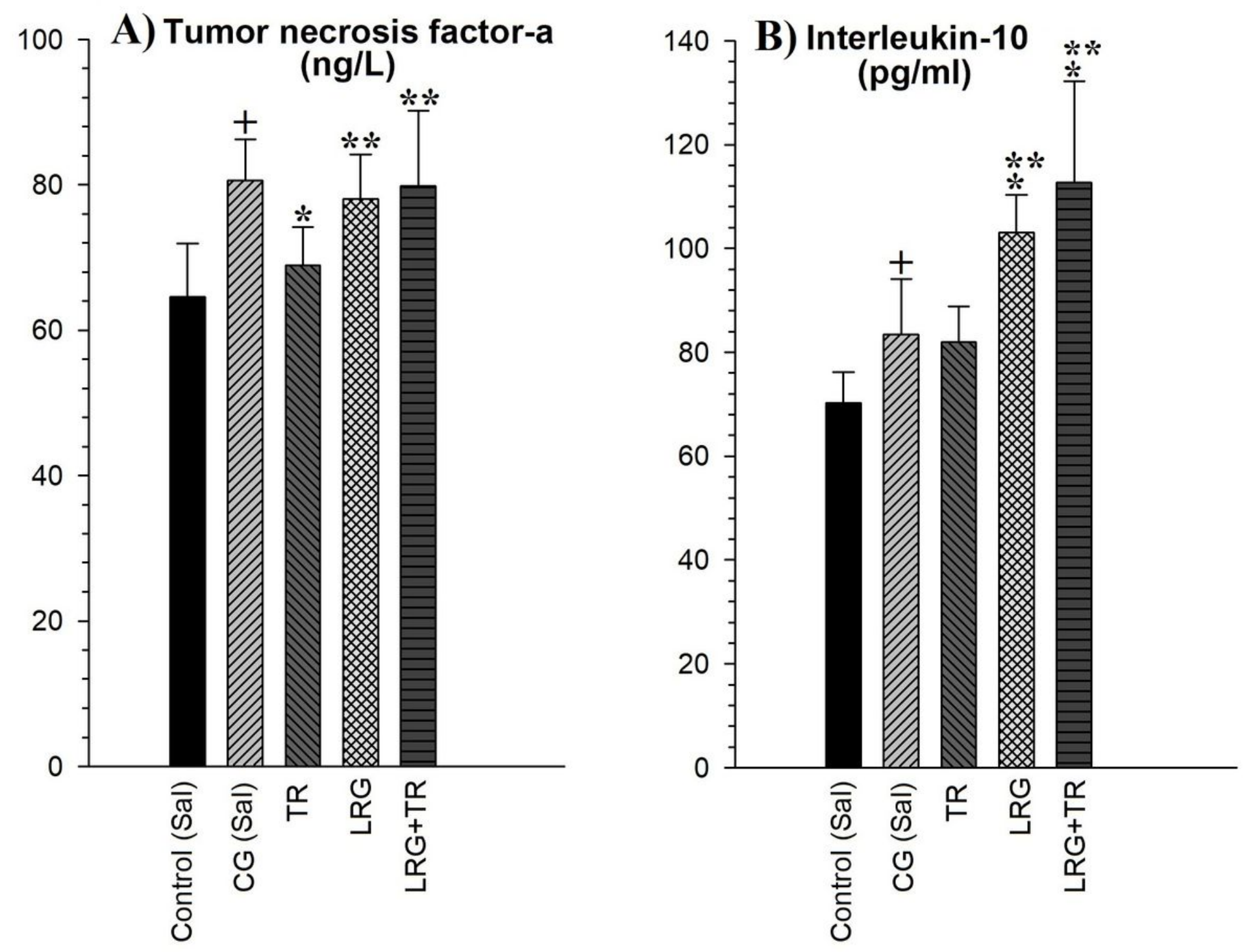

Figure 5

Effects of Tramadol (TR), Liraglutide (LRG) or their combination (TR+LRG) on tumor necrosis factor alpha (TNF-a) (A) and interleukin (IL)-10 (B) in carrageenan (CG) induced inflamed paws of rats. Each bar represents the mean value of 6 rats, and the vertical bars indicate \pm SD. $+P<0.05$ indicates significant differences as compared to control (saline) group. ${ }^{*} P<0.05$ indicates significant differences as compared to CG-control group. ** $P<0.05$ indicates significant differences as compared to TR group 


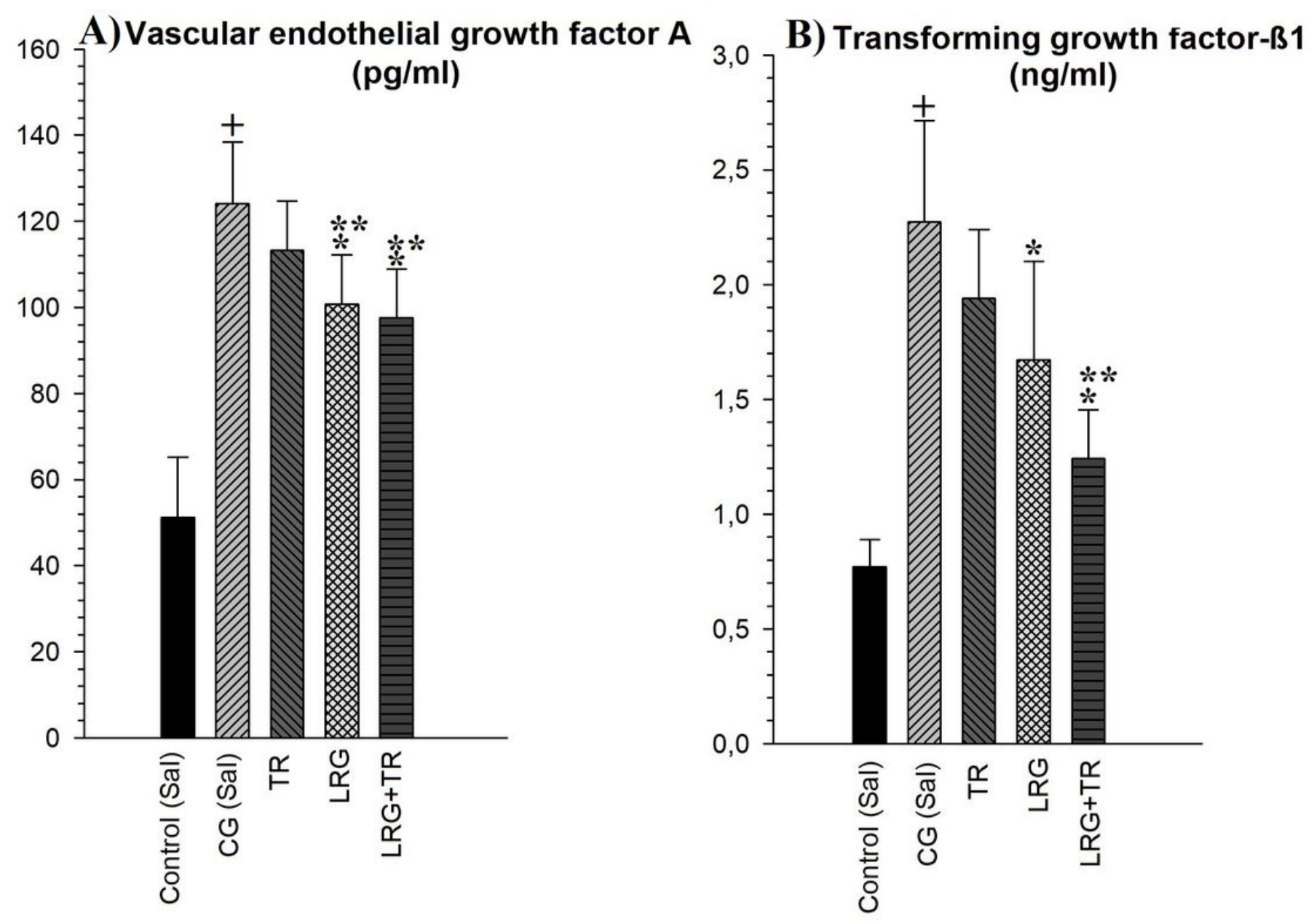

Figure 6

Effects of Tramadol (TR), Liraglutide (LRG) or their combination (TR+LRG) on vascular endothelial growth factor A (VEGF-A) (A) and transforming growth factor $\beta 1$ (B) in carrageenan (CG) induced inflamed paws of rats. Each bar represents the mean value of 6 rats, and the vertical bars indicate \pm SD. + $P<0.05$ indicates significant differences as compared to control (saline) group. * $P<0.05$ indicates significant differences as compared to CG-control group. ${ }^{*} \mathrm{P}<0.05$ indicates significant differences as compared to TR group 


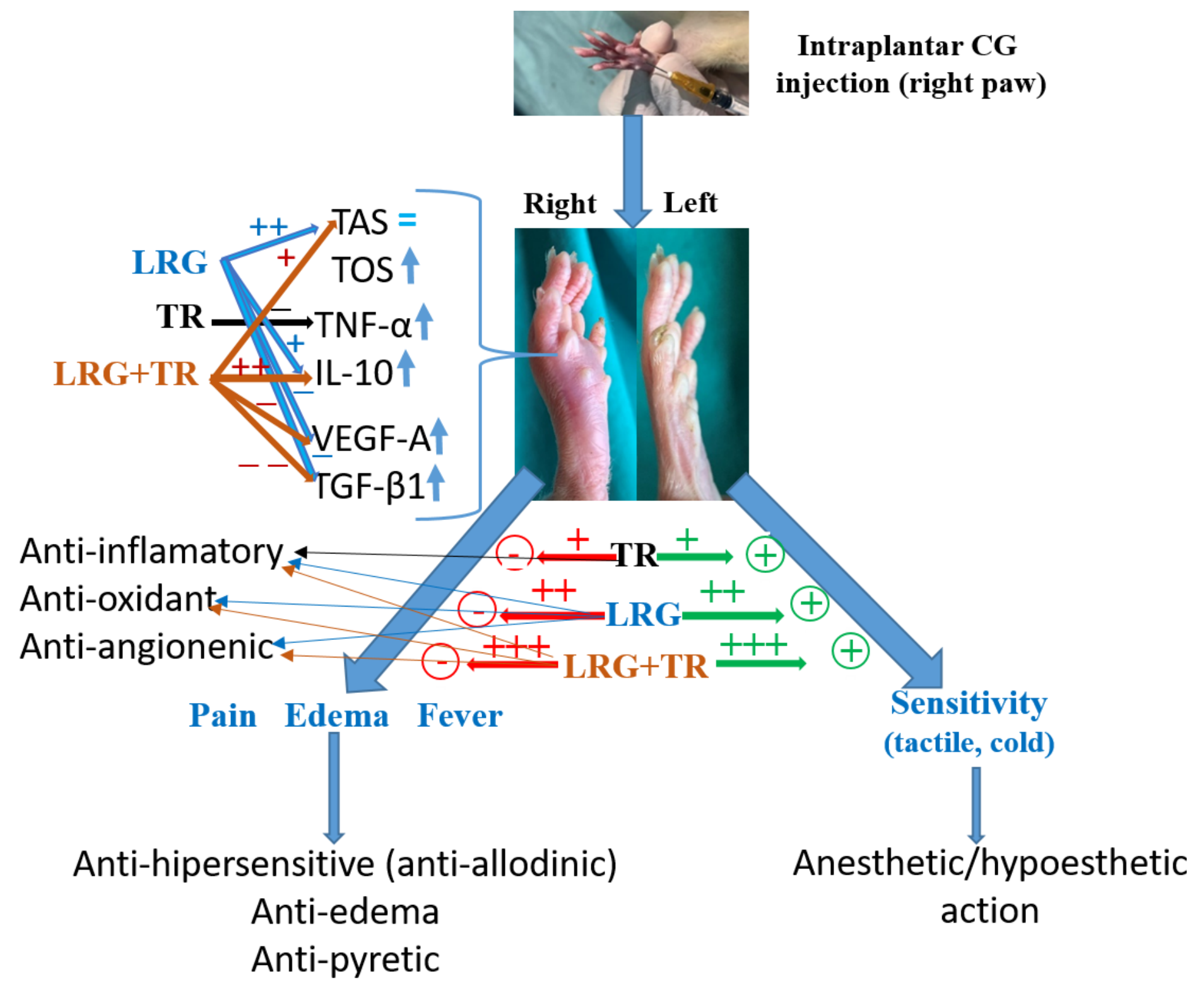

Figure 7

Schematic representation of possible action mechanisms of Tramadol (TR), Liraglutide (LRG) or their combination (TR+LRG) in carrageenan (CG) induced inflamed paws of rats 